\title{
All-Optical Initialization, Readout, and Coherent Preparation of Single Silicon-Vacancy Spins in Diamond
}

\author{
Lachlan J. Rogers, ${ }^{1}$ Kay D. Jahnke, ${ }^{1}$ Mathias H. Metsch, ${ }^{1}$ Alp Sipahigil, ${ }^{2}$ Jan M. Binder, ${ }^{1}$ Tokuyuki Teraji, ${ }^{3}$ \\ Hitoshi Sumiya, ${ }^{4}$ Junichi Isoya, ${ }^{5}$ Mikhail D. Lukin, ${ }^{2}$ Philip Hemmer, ${ }^{6}$ and Fedor Jelezko ${ }^{1}$ \\ ${ }^{1}$ Institute for Quantum Optics and Center for Integrated Quantum Science and Technology (IQst), \\ Ulm University, Ulm D-89081, Germany \\ ${ }^{2}$ Department of Physics, Harvard University, 17 Oxford Street, Cambridge, Massachusetts 02138, USA \\ ${ }^{3}$ National Institute for Materials Science, 1-1 Namiki, Tsukuba, Ibaraki 305-0044, Japan \\ ${ }^{4}$ Advanced Materials $R$ \& D Laboratories, Sumitomo Electric Industries Limited, Itami, Hyogo 664-0016, Japan \\ ${ }^{5}$ Research Center for Knowledge Communities, University of Tsukuba, 1-2 Kasuga, Tsukuba, Ibaraki 305-8550, Japan \\ ${ }^{6}$ Electrical \& Computer Engineering Department, Texas A\&M University, College Station, Texas 77843, USA
}

(Received 12 October 2014; published 22 December 2014)

\begin{abstract}
The silicon-vacancy $\left(\mathrm{SiV}^{-}\right)$color center in diamond has attracted attention because of its unique optical properties. It exhibits spectral stability and indistinguishability that facilitate efficient generation of photons capable of demonstrating quantum interference. Here we show optical initialization and readout of electronic spin in a single $\mathrm{SiV}^{-}$center with a spin relaxation time of $T_{1}=2.4 \pm 0.2 \mathrm{~ms}$. Coherent population trapping (CPT) is used to demonstrate coherent preparation of dark superposition states with a spin coherence time of $T_{2}^{\star}=35 \pm 3 \mathrm{~ns}$. This is fundamentally limited by orbital relaxation, and an understanding of this process opens the way to extend coherence by engineering interactions with phonons. Hyperfine structure is observed in CPT measurements with the ${ }^{29} \mathrm{Si}$ isotope which allows access to nuclear spin. These results establish the $\mathrm{SiV}^{-}$center as a solid-state spin-photon interface.
\end{abstract}

Coherent quantum systems which efficiently couple long-lived quantum memories to optical photons are a key resource for realizing quantum networks [1]. Color centers in diamond [2] are attractive candidates owing to unique properties of diamond, which include optical transparency and a high lattice quality that allows spin to function as long-lived quantum memory [3]. The negative silicon-vacancy $\left(\mathrm{SiV}^{-}\right)$defect in diamond [4-6] has exceptional optical properties that facilitate efficient generation of indistinguishable photons from multiple distinct emitters [7]. Here we show optical initialization and readout of electronic spin in a single $\mathrm{SiV}^{-}$center with a spin relaxation time of $T_{1}=2.4 \pm 0.2 \mathrm{~ms}$. Two-photon resonance [8] is used to demonstrate coherent preparation of dark superposition states with a spin coherence time of $T_{2}^{\star}=35 \pm 3 \mathrm{~ns}$. This is shown to be limited by orbital relaxation that may be suppressed by engineering interactions with phonons. We present the first evidence of hyperfine interaction with a ${ }^{29} \mathrm{Si}$ nuclear spin in $\mathrm{SiV}^{-}$which can potentially be used as a memory qubit [9].

Quantum information processing efforts in diamond have mainly focused on the nitrogen-vacancy $\left(\mathrm{NV}^{-}\right)$center because of its excellent spin properties at ambient conditions

Published by the American Physical Society under the terms of the Creative Commons Attribution 3.0 License. Further distribution of this work must maintain attribution to the author(s) and the published article's title, journal citation, and DOI.
[10]. All-optical access to $\mathrm{NV}^{-}$spin is possible [11-14]; however, its large phonon sideband and spectral diffusion reduce coherent photon generation rates and limit the development of $\mathrm{NV}^{-}$quantum networks [15-17]. The main optical advantage provided by the $\mathrm{SiV}^{-}$center is that $70 \%$ of its fluorescence is concentrated in a sharp zero-phonon line (ZPL), making it ideal for single photon source applications $[18,19]$. It is spectrally stable at $737 \mathrm{~nm}$, exhibits line widths limited by the excited state lifetime [20], and can be coupled to optical cavities $[21,22]$. Physically, the $\mathrm{SiV}^{-}$center consists of a single silicon atom replacing two carbon atoms in the diamond lattice, forming $\mathrm{D}_{3 \mathrm{~d}}$ symmetry as illustrated in Fig. 1(a) [4-6,23]. This geometry makes the $\mathrm{SiV}^{-}$center insensitive to small electric fields [7] and therefore adds low inhomogeneous broadening to the set of attractive optical properties. The electronic structure of the $\mathrm{SiV}^{-}$center consists of doubly degenerate $\mathrm{E}$ ground and excited state orbitals which both have electronic spin-1/2 [4-6]. Optical signatures of the electron spin have been identified [24]; however, control of $\mathrm{SiV}^{-}$spin remains an outstanding challenge.

The technique used here to manipulate and measure $\mathrm{SiV}^{-}$ spin involves resonant excitation with narrow-band lasers. A diamond plate with $\{111\}$ surfaces (see Ref. [25] for details of the synthesis) was cooled to around $5 \mathrm{~K}$ in a continuous flow cryostat, and imaged using a home-built confocal fluorescence microscope. Resonant excitation spectra of the four fine-structure transitions A-D [Fig. 1(b)] were measured for grown-in $\mathrm{SiV}^{-}$centers by scanning a high-resolution $(100 \mathrm{kHz})$ resonant laser and recording sideband fluorescence 
(a)

(c)

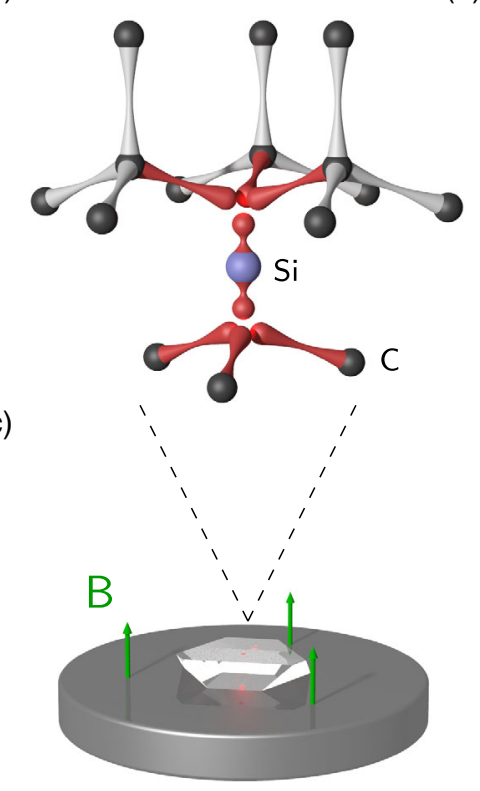

(b)
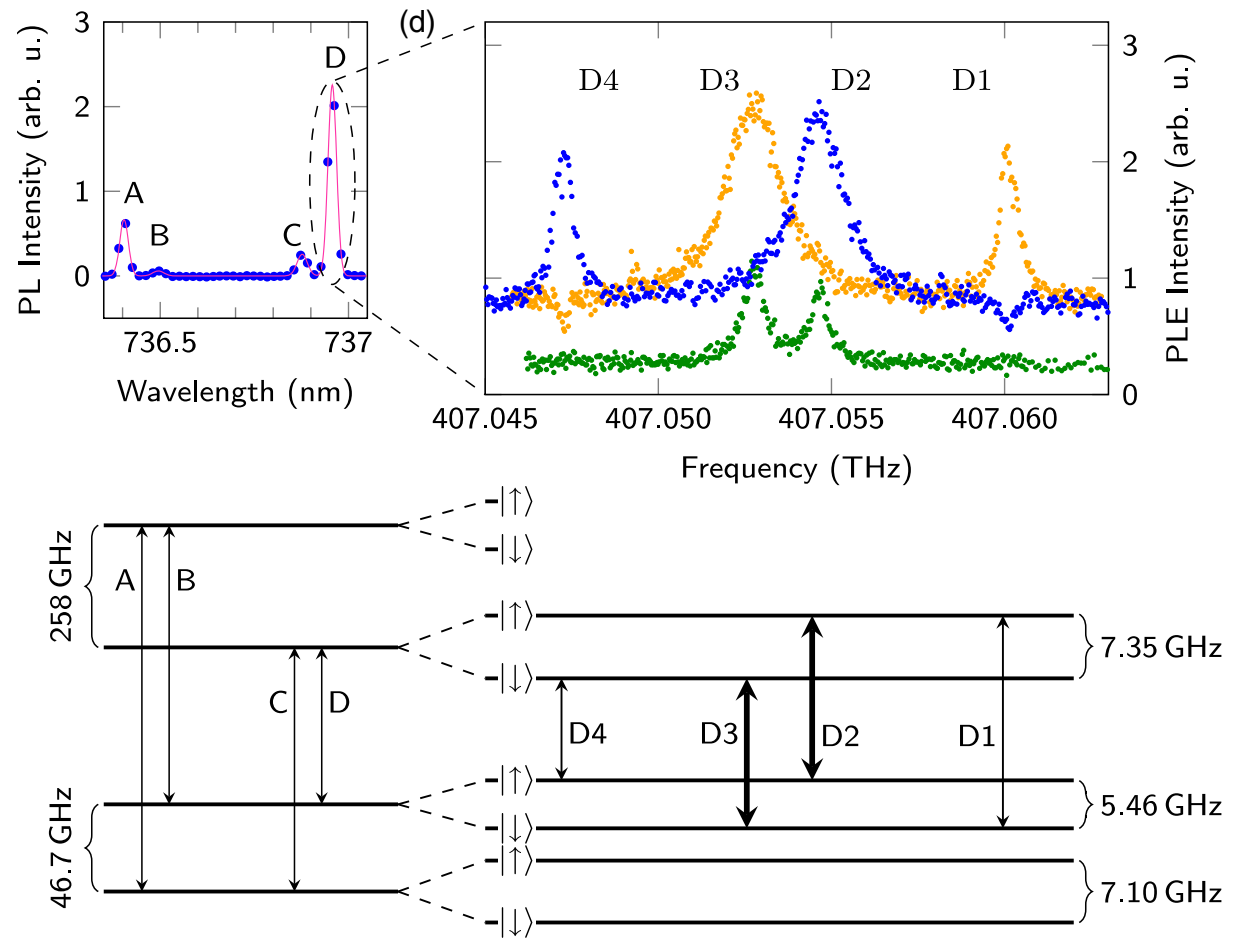

FIG. 1 (color online). Optical access to spin levels of $\mathrm{SiV}^{-}$in diamond. (a) $\mathrm{A} \mathrm{SiV}^{-}$center consists of a silicon atom between two vacant lattice sites and is aligned along a $\langle 111\rangle$ crystal bond direction. (b) At zero field the ZPL consists of four transitions, which occur between spin-orbit branches of the doublet ground and excited states as illustrated below the spectrum. Transitions B and C appear weak since the $\mathrm{SiV}^{-}$is viewed here along its symmetry axis. (c) Zeeman splitting of the spin- $1 / 2$ electronic states $(|\uparrow\rangle$ and $|\downarrow\rangle$ for simplicity) was achieved by mounting the diamond on a neodymium magnet. This produced a field around $4.5 \mathrm{kG}$ closely aligned with the $\mathrm{SiV}^{-}$centers oriented normal to the $\{111\}$ sample surface. (d) Resonant excitation spectroscopy using a single laser reveals the two spin-conserving transitions D2 and D3 (green). Applying a second pump laser on the D2 (orange) or D3 (blue) transition makes the spinflipping transitions D1 and D4 appear in the excitation spectrum. These optical transitions between the Zeeman-split levels are illustrated beneath the spectrum (bold indicates spin conserving).

through a 750-810 $\mathrm{nm}$ band-pass filter. Mounting the diamond on a fixed magnet as shown in Fig. 1(c) lifted the spin degeneracy by Zeeman splitting, and excitation spectra were recorded for $\mathrm{a} \mathrm{SiV}^{-}$site aligned to a $\sim 4.5 \mathrm{kG}$ magnetic field. This optical alignment obscures the axial transition dipole, making transition $\mathrm{D}$ the brightest and narrowest peak $[6,20]$. It split into two subpeaks as shown in Fig. 1(d), and two additional peaks were observed when a second laser was pumping either of the two bright transitions. These four spectral features correspond to the four possible transitions between the Zeeman-split electronic spin sublevels of the orbital branches involved in transition D (numbered D1-D4 with decreasing energy). A qualitatively identical pattern of four features was measured for transition C [25], allowing a determination of the Zeeman splittings illustrated in Fig. 1(d).

Although optical transitions are spin conserving, any offaxis magnetic field results in a different quantization axis between the ground and the excited states because of the difference in spin-orbit couplings [5]. In our experiments, a residual off-axis field makes the spin-flipping transitions (D1 and D4) weakly allowed. These transitions therefore pump the spin into a dark state after a few optical cycles and are not visible in single laser scans. Figure 2(a) shows time-resolved fluorescence measurements where we utilized the D1 transition to optically pump the spin into a dark state and the cycling D2 transition to efficiently read out the resulting spin state. Laser pulses were produced using acousto-optical modulators, giving a rise or fall time of about 60 ns but a constant intensity over the duration of the pulse. The fluorescence produced by the D2 transition had a prominent leading edge when the interpulse delay was small, which is evidence of optical pumping causing spin initialization. The decay of the leading-edge height $h$ with increasing pulse separation $\tau$ establishes the spin relaxation time in the aligned field at $T_{1}^{\mathrm{spin}}=2.4 \pm 0.2 \mathrm{~ms}$, as shown in Fig. 2(b).

For a $\mathrm{SiV}^{-}$center misaligned about 20 degrees from the magnetic field, the spin relaxation time was measured to be $T_{1}^{\text {sin }}=3.4 \mu \mathrm{s}$, and a 70-degree misalignment reduced this to $T_{1}^{\text {spin }} \sim 60 \mathrm{~ns}$ [25]. This sharp decrease in spin relaxation time is a direct result of the spin state mixing induced by the off-axis magnetic field, and is accompanied by peaks D1 and D4 starting to appear in excitation spectra without the need for a second repumping laser. Given the weak D1 transition dipole for aligned magnetic fields, the measured $78 \%$ spin initialization fidelity [Fig. 2(a)] was limited by laser intensity due to competition between the long spin relaxation and slow optical pumping rates on D1. Equivalent measurements with the field misaligned by about 20 degrees, where the 
(a)

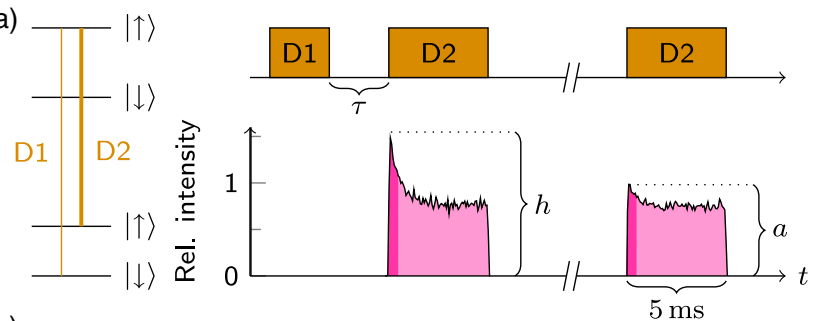

(b)

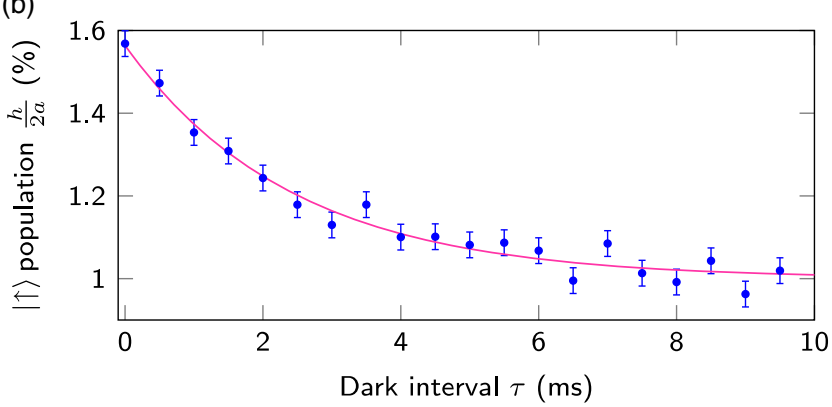

(c)

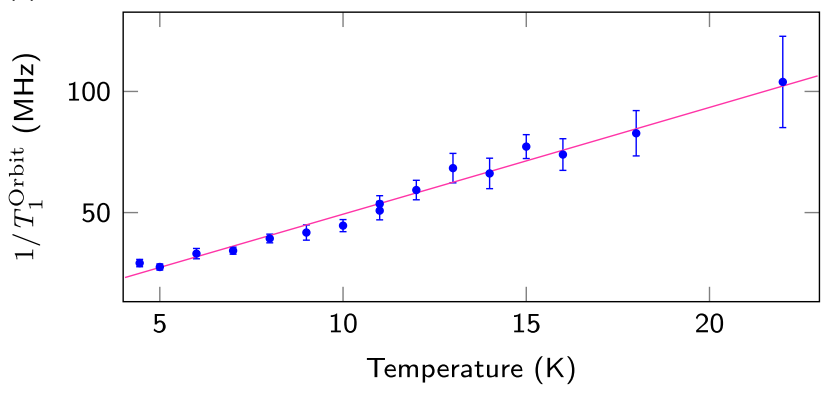

FIG. 2 (color online). Optical initialization and readout of $\mathrm{SiV}^{-}$ spin. (a) A laser pulse resonant to transition D1 flips the spin without producing measurable fluorescence. The initialized spin state can be read out using a laser pulse on the cycling transition D2. The spin polarization signal appears as a leading-edge peak of the fluorescence that decays in about $500 \mu \mathrm{s}$, here shaded darker, which contained on average one photon per pulse. After a long dark interval $\tau$ the peak height reduces to an asymptotic value $a$. (b) The reduction of $h$ with increasing $\tau$ gives the spin relaxation time at $T_{1}^{\text {spin }}=2.4 \pm 0.2 \mathrm{~ms}$. Interpreting the asymptotic limit $a$ to correspond to thermal spin population suggests a spin initialization fidelity of $h_{\tau=0} / 2 a=78 \%$. (c) Similar pulsed measurements give the orbital relaxation time at $4.5 \mathrm{~K}$ as $T_{1}^{\text {Orbit }}=38 \pm 1 \mathrm{~ns}$. The orbital relaxation rate $1 / T_{1}^{\text {Orbit }}$ increased linearly with temperature.

spin-flipping transition is stronger, demonstrated an initialization fidelity of at least $95 \%$ [25].

These observations indicate that the $\mathrm{SiV}^{-}$center provides robust access to both spin-flipping lambda transitions and long-cycling spin-conserving transitions which enable optical readout of the spin state. During each D2 laser pulse shown in Fig. 2, the fluorescence decayed from its initial height $h$ in about $500 \mu$ s. This shaded readout interval is long given that the D2 transition was excited above saturation, indicating the cyclicity of this transition. Such long-cycling transitions should allow single-shot spin readout with high fidelity depending primarily on photon collection efficiency [27].

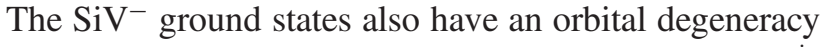
which can introduce additional dynamics beyond $\operatorname{spin} T_{1}^{\text {spin }}$ processes $[28,29]$. Orbital relaxation was isolated from any spin pumping/relaxation by measuring at zero magnetic field. Since the time scale was found to be fast compared to the acousto-optical modulation bandwidth, laser pulses of $80 \mathrm{~ns}$ (1 ns rise/fall time) were produced using an elecro-optical modulator and applied resonant to transition $\mathrm{D}$. Photon counts were recorded with timing resolution of $200 \mathrm{ps}$, and a leadingedge peak similar to that in Fig. 2(a) was observed. In this case the decay of the initial intensity corresponds to population being pumped to the other ground-state branch (decaying via transition C). Varying the dark interval between subsequent pulses revealed that the leading-edge peak recovered in an orbital relaxation time of only $T_{1}^{\text {Orbit }}=38 \pm 1 \mathrm{~ns}$ at $4.5 \mathrm{~K}$. This is much shorter than $T_{1}^{\text {spin }}$, which indicates that the orbital relaxation must be highly spin conserving, as expected for electron-phonon interactions. Measurements of $T_{1}^{\text {Orbit }}$ were repeated for temperatures up to $22 \mathrm{~K}$, and the rate was found to vary linearly with temperature [Fig. 2(c)]. This is evidence of a single-phonon mechanism for this fast relaxation process [30-32], which has significant implications for the spin coherence time discussed below.

With this understanding of the transitions and the population dynamics, it is possible to identify $\Lambda$ schemes consisting of two allowed transitions from different ground states to a common excited state. Such $\Lambda$ schemes provide the ability to optically prepare a dark superposition of the ground states [8]. The dark state causes a dip in the excitation spectrum with a width limited by the phase lifetime of the superposition. This phenomenon is known as coherent population trapping (CPT) and is used here to probe ground-state spin coherence.

To observe CPT, a pump laser was tuned to transition D2 and a probe laser was scanned across transition D1. The excitation peak for transition D1 was observed to contain a sharp two-photon resonance dip as shown in Fig. 3(a). The minimum width of this dip was probed by generating both excitation frequencies from a single laser, using a high bandwidth electro-optic amplitude modulator operated in the linear response regime. The modulation frequency was scanned across the two-photon detuning using microwave sources, and the CPT linewidths in Figs. 3(b) and 3(c) are therefore insensitive to laser frequency noise. Laser power limitations with this equipment prevented access to the dark transitions for the well-aligned field, and so measurements were made on a second $\mathrm{SiV}^{-}$site oriented at about 70 degrees to the field, which accounts for the lower D1-D2 detuning. The CPT dip width was measured for various laser powers [Fig. 3(b)] and was found to be about $4.5 \pm 0.3 \mathrm{MHz}$ in the absence of power broadening [Fig. 3(c)]. This is far below the transform-limited optical linewidth of $94 \mathrm{MHz}$ [20], which confirms CPT and corresponds to a spin coherence time of $T_{2}^{\star}=35 \pm 3$ ns. This coherence time is similar to $T_{1}^{\text {Orbit }}$, indicating that the rapid switching between orbital branches leads to dephasing of the electronic spin even when its polarization is maintained. Although this dramatically limits the usefulness of electronic spin in $\mathrm{SiV}^{-}$, our identification of 
(a)

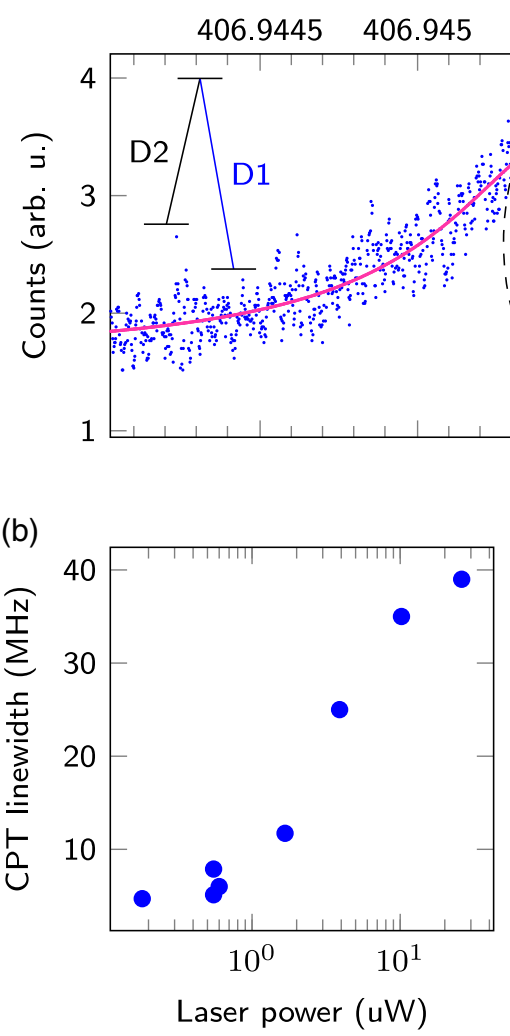

Probe laser frequency $(\mathrm{THz})$

$\begin{array}{llll}406.9455 & 406.946 & 406.9465 & 406.947\end{array}$

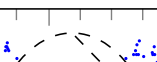

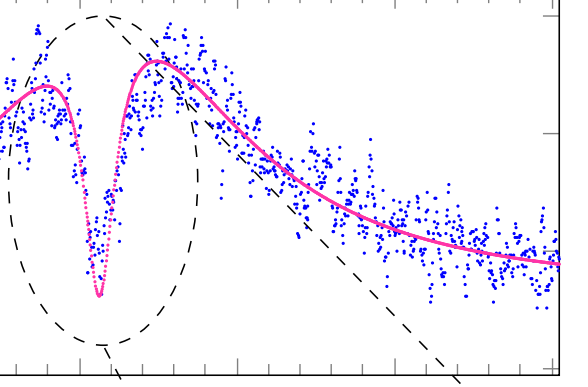

(c)

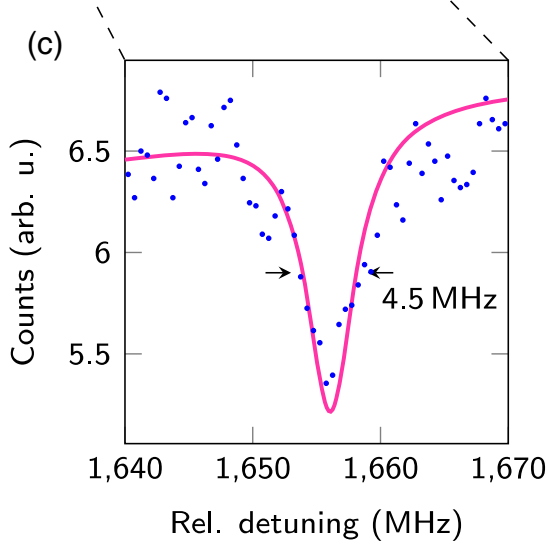

(d)

Rel. detuning $(\mathrm{GHz})$
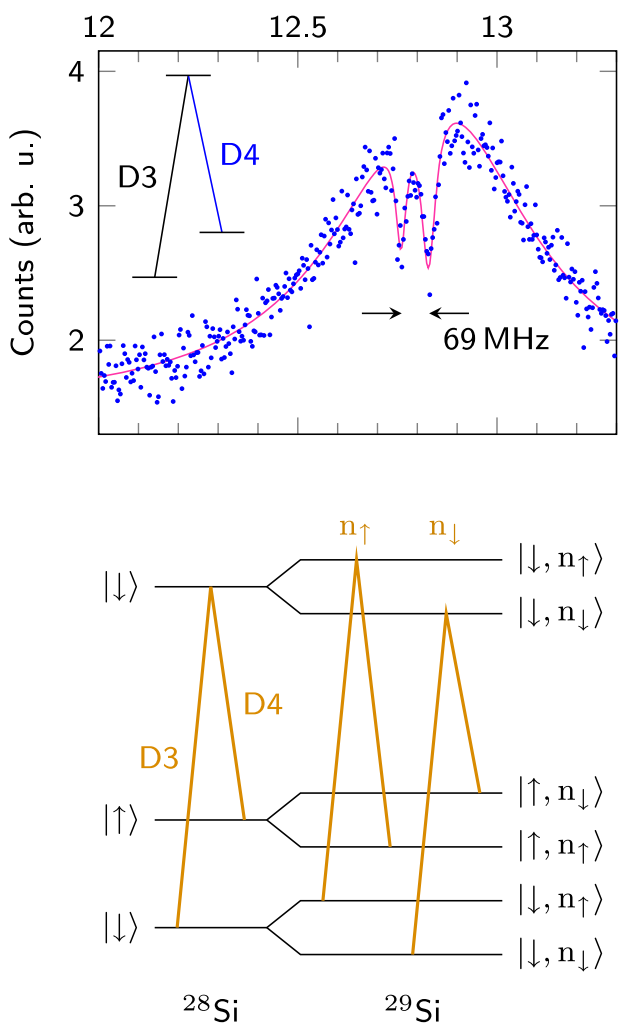

FIG. 3 (color online). Preparation of coherent dark states. (a) Typical excitation spectrum for a probe laser scanned across transition D1 while a second pump laser was applied resonant to D2. These transitions form a $\Lambda$ scheme giving rise to CPT, producing the characteristic sharp dip in the spectrum. (b) Reducing the incident laser powers made it possible to avoid power broadening of the CPT dip. (c) The narrowest dip FWHM was $4.5 \pm 0.3 \mathrm{MHz}$, corresponding to a spin coherence time of $T_{2}^{\star}=35 \pm 3 \mathrm{~ns}$. (d) A double CPT dip was observed for $\mathrm{SiV}^{-}$centers containing the isotope ${ }^{29} \mathrm{Si}$ which has nuclear spin $I=\frac{1}{2}$. The nuclear Zeeman shift is reversed for the opposite electronic spin, and so the two nuclear-spin-conserving $\Lambda$ schemes occur at different relative laser detunings. The splitting of $69 \mathrm{MHz}$ between the CPT dips suggests a nuclear hyperfine splitting on the order of $35 \mathrm{MHz}$. In order to more clearly resolve the nuclear splitting in Fig. 3(d), a stronger magnetic field was applied by mounting the sample on a pattern of four cubic magnets. This accounts for the increased relative detuning between transitions D3 and D4.

the orbital switching mechanism makes it possible to consider techniques to overcome this limit.

One approach to create a long-lived memory is provided by $\mathrm{SiV}^{-}$centers containing the ${ }^{29} \mathrm{Si}$ isotope, which has nuclear spin- $\frac{1}{2}$. Such $\mathrm{SiV}^{-}$centers can be identified by an isotopic shift of the ZPL [23]. We observed a double CPT dip for ${ }^{29} \mathrm{Si}$ $\mathrm{SiV}^{-}$sites, as shown in Fig. 3(c). This doublet arises because of the hyperfine interaction with the ${ }^{29} \mathrm{Si}$ nuclear spin $(I=1 / 2)$ which gives rise to a two-photon resonance condition at two different detunings, as shown in Fig. 3(d). This observation of ${ }^{29} \mathrm{Si}$ hyperfine splitting in $\mathrm{SiV}^{-}$raises the possibility of optical access to nuclear spin, which should have much longer coherence times than electron spins [33-35].

It may also be possible to improve the coherence time of electronic spin in $\mathrm{SiV}^{-}$. Since $T_{2}^{\star}$ is limited by orbital relaxation that arises from single-phonon processes, most of these ideas revolve around limiting the availability of phonons at the relevant frequency (around $47 \mathrm{GHz}$ ). For temperatures below $47 \mathrm{GHz}(\ll 2 \mathrm{~K})$, the reduced phonon occupation results in a suppression of the orbital relaxation from the lower branch of the ground state. Using the lower branch states as qubits would then enable long-lived spin coherences. Even at $5 \mathrm{~K}$, nanodiamonds with sizes smaller than half the transition phonon wavelength (around $125 \mathrm{~nm}$ ) would inhibit the orbital relaxation rate [36], as the diamondvacuum interface results in a $3 \mathrm{D}$ phonon cavity with a discrete spectrum [37]. Similarly, recent advances in diamond nanofabrication $[38,39]$ suggest that periodic nanostructures can be fabricated on diamond to realize a complete phononic band gap at the phonon transition frequency [40-42]. We expect both approaches to reduce the orbital relaxation rate and therefore increase the spin coherence time.

We have demonstrated that resonant excitation can be used to read out and coherently prepare electronic spin in individual $\mathrm{SiV}^{-}$centers. These results open the door for exploration of long-lived quantum memories based on $\mathrm{SiV}^{-}$centers and the development of spin-photon interfaces benefiting from unique optical properties.

We acknowledge ERC, EU projects (SIQS, DIADEMS, EQUAM), DFG (FOR 1482, FOR 1493 and SFBTR 21), JST, JSPS KAKENHI (No. 26246001), BMBF, USARL/ORISE, 
DARPA (QuASAR, QUINESS), NSF, CUA, CIQM, and the Sino-German and Volkswagen foundations for funding. L. R., M. M., K. J., J. B., and A.S. performed the experiments, which were conceived by P. H., F. J., and M. L.. T. T., H. S., and J. I. synthesized the samples. L. R., K. J., and M. M. wrote the manuscript with input from all the authors.

Note added.-Recently, we became aware of complementary work reporting CPT with $\mathrm{SiV}^{-}$centers [43].

[1] H. J. Kimble, Nature (London) 453, 1023 (2008).

[2] I. Aharonovich and E. Neu, Adv. Opt. Mater. 2, 911 (2014).

[3] G. Balasubramanian, P. Neumann, D. Twitchen, M. Markham, R. Kolesov, N. Mizuochi, J. Isoya, J. Achard, J. Beck, J. Tissler, V. Jacques, P. R. Hemmer, F. Jelezko, and J. Wrachtrup, Nat. Mater. 8, 383 (2009).

[4] J. P. Goss, R. Jones, S. J. Breuer, P. R. Briddon, and S. Öberg, Phys. Rev. Lett. 77, 3041 (1996).

[5] C. Hepp, T. Müller, V. Waselowski, J. N. Becker, B. Pingault, H. Sternschulte, D. Steinmüller-Nethl, A. Gali, J. R. Maze, M. Atatüre, and C. Becher, Phys. Rev. Lett. 112, 036405 (2014).

[6] L. J. Rogers, K. D. Jahnke, M. W. Doherty, A. Dietrich, L. P. McGuinness, C. Müller, T. Teraji, H. Sumiya, J. Isoya, N. B. Manson, and F. Jelezko, Phys. Rev. B 89, 235101 (2014).

[7] A. Sipahigil, K. D. Jahnke, L. J. Rogers, T. Teraji, J. Isoya, A. S. Zibrov, F. Jelezko, and M. D. Lukin, Phys. Rev. Lett. 113, 113602 (2014).

[8] M. Fleischhauer, A. Imamoglu, and J. P. Marangos, Rev. Mod. Phys. 77, 633 (2005).

[9] E. Togan, Y. Chu, A. Imamoglu, and M. D. Lukin, Nature (London) 478, 497 (2011).

[10] L. Childress, R. Walsworth, and M. Lukin, Phys. Today 67, No. 10, 38 (2014).

[11] C. Santori, P. Tamarat, P. Neumann, J. Wrachtrup, D. Fattal, R. G. Beausoleil, J. Rabeau, P. Olivero, A. D. Greentree, S. Prawer, F. Jelezko, and P. R. Hemmer, Phys. Rev. Lett. 97, 247401 (2006).

[12] C. Santori, D. Fattal, S. M. Spillane, M. Fiorentino, R. G. Beausoleil, A. D. Greentree, P. Olivero, M. Draganski, J. R. Rabeau, P. Reichart, B. C. Gibson, S. Rubanov, D. N. Jamieson, and S. Prawer, Opt. Express 14, 7986 (2006).

[13] B. B. Buckley, G. D. Fuchs, L.C. Bassett, and D. D. Awschalom, Science 330, 1212 (2010).

[14] L. C. Bassett, F. J. Heremans, D. J. Christle, C. G. Yale, G. Burkard, B. B. Buckley, and D. D. Awschalom, Science 345, 1333 (2014).

[15] H. Bernien, B. Hensen, W. Pfaff, G. Koolstra, M. S. Blok, L. Robledo, T. H. Taminiau, M. Markham, D. J. Twitchen, L. Childress, and R. Hanson, Nature (London) 497, 86 (2013).

[16] H. Bernien, L. Childress, L. Robledo, M. Markham, D. Twitchen, and R. Hanson, Phys. Rev. Lett. 108, 043604 (2012).

[17] A. Sipahigil, M. L. Goldman, E. Togan, Y. Chu, M. Markham, D. J. Twitchen, A. S. Zibrov, A. Kubanek, and M. D. Lukin, Phys. Rev. Lett. 108, 143601 (2012).

[18] E. Neu, D. Steinmetz, J. Riedrich-Möller, S. Gsell, M. Fischer, M. Schreck, and C. Becher, New J. Phys. 13, 025012 (2011).

[19] M. Leifgen, T. Schröder, F. Gädeke, R. Riemann, V. Métillon, E. Neu, C. Hepp, C. Arend, C. Becher, K. Lauritsen, and O. Benson, New J. Phys. 16, 023021 (2014).
[20] L. J. Rogers, K. D. Jahnke, T. Teraji, L. Marseglia, C. Müller, B. Naydenov, H. Schauffert, C. Kranz, J. Isoya, L.P. McGuinness, and F. Jelezko, Nat. Commun. 5, 4739 (2014).

[21] J. C. Lee, I. Aharonovich, A. P. Magyar, F. Rol, and E. L. Hu, Opt. Express 20, 8891 (2012).

[22] J. Riedrich-Möller, C. Arend, C. Pauly, F. Mücklich, M. Fischer, S. Gsell, M. Schreck, and C. Becher, Nano Lett. 14, 5281 (2014).

[23] A. Dietrich, K. D. Jahnke, J. M. Binder, T. Teraji, J. Isoya, L. J. Rogers, and F. Jelezko New J. Phys. 16, 113019 (2014).

[24] T. Müller, C. Hepp, B. Pingault, E. Neu, S. Gsell, M. Schreck, H. Sternschulte, D. Steinmüller-Nethl, C. Becher, and M. Atatüre, Nat. Commun. 5, 3328 (2014).

[25] See Supplemental Material at http://link.aps.org/ supplemental/10.1103/PhysRevLett.113.263602, which includes Ref. [26], for details on sample synthesis, transitions $C 1-C 4$, population dynamics, spin relaxation in misaligned field, and CPT on an orbital $\Lambda$ scheme.

[26] H. Sumiya and K. Tamasaku, Jpn. J. Appl. Phys. 51, 090102 (2012).

[27] L. Robledo, L. Childress, H. Bernien, B. Hensen, P. F. A. Alkemade, and R. Hanson, Nature (London) 477, 574 (2011).

[28] A. M. Stoneham, Theory of Defects in Solids: Electronic Structure of Defects in Insulators and Semiconductors (Oxford University Press, New York, 2001).

[29] F. S. Ham, Phys. Rev. 138, A1727 (1965).

[30] R. Orbach, Proc. R. Soc. A 264, 458 (1961).

[31] P. L. Scott and C. D. Jeffries, Phys. Rev. 127, 32 (1962).

[32] K. D. Jahnke, A. Sipahigil, J. M. Binder, M. W. Doherty, M. Metsch, L. J. Rogers, N. B. Manson, M. D. Lukin, and F. Jelezko, arXiv:1411.2871.

[33] L. Childress, M. V. G. Dutt, J. M. Taylor, A. S. Zibrov, F. Jelezko, J. Wrachtrup, P. R. Hemmer, and A. M. D. Lukin, Science 314, 281 (2006).

[34] V. Jacques, P. Neumann, J. Beck, M. Markham, D. Twitchen, J. Meijer, F. Kaiser, G. Balasubramanian, F. Jelezko, and J. Wrachtrup, Phys. Rev. Lett. 102, 057403 (2009).

[35] P. C. Maurer, G. Kucsko, C. Latta, L. Jiang, N. Y. Yao, S. D. Bennett, F. Pastawski, D. Hunger, N. Chisholm, M. Markham, D. J. Twitchen, J. I. Cirac, and M. D. Lukin, Science 336, 1283 (2012).

[36] D. Kleppner, Phys. Rev. Lett. 47, 233 (1981).

[37] A. Albrecht, A. Retzker, F. Jelezko, and M. B. Plenio, New J. Phys. 15, 083014 (2013).

[38] Y. Tao, J. M. Boss, B. A. Moores, and C. L. Degen, Nat. Commun. 5, 3638 (2014).

[39] M. J. Burek, D. Ramos, P. Patel, I. W. Frank, and M. Lončar, Appl. Phys. Lett. 103, 131904 (2013).

[40] A. H. Safavi-Naeini, J. T. Hill, S. Meenehan, J. Chan, S. Gröblacher, and O. Painter, Phys. Rev. Lett. 112, 153603 (2014).

[41] J. Gomis-Bresco, D. Navarro-Urrios, M. Oudich, S. ElJallal, A. Griol, D. Puerto, E. Chavez, Y. Pennec, B. DjafariRouhani, F. Alzina, A. Martínez, and C. M. S. Torres, Nat. Commun. 5, 4452 (2014).

[42] L. Kipfstuhl, F. Guldner, J. Riedrich-Möller, and C. Becher, Opt. Express 22, 12410 (2014).

[43] B. Pingault, J. N. Becker, C. H. H. Schulte, C. Arend, C. Hepp, T. Godde, A. I. Tartakovskii, M. Markham, C. Becher, and M. Atature, following Letter, Phys. Rev. Lett. 113, 263601 (2014). 